\title{
Materialização de questões relacionadas a Centro de Massa: uma atividade aplicada a licenciandos
}

Adriana da Silva Fontes asfontes@utfpr.edu.br

Universidade Tecnológica Federal do Paraná (UTFPR), Campo Mourão,

Paraná, Brasil.

Fernanda Peres Ramos fernandaramos@uttpr.edu.br Universidade Tecnológica Federal do Paraná (UTFPR), Campo Mourão, Paraná, Brasil.

Cesar Vanderlei Deimling cdeimling@gmail.com

Universidade Tecnológica Federal do

Paraná (UTFPR), Campo Mourão,

Paraná, Brasil.

\section{RESUM 0}

Apresenta-se, por meio desse artigo, uma pesquisa ação desenvolvida com licenciandos do curso de Química em uma Universidade Pública no interior do Paraná, na disciplina de Física 1. Buscou-se, com essa atividade, contribuir para minimizar a dificuldade dos acadêmicos na interpretação de exercícios do livro texto adotado, por meio da materialização de exercícios sobre o tema Centro de massa. Inicialmente o conteúdo foi introduzido de modo a identificar o conhecimento prévio. Na sequência trabalhou-se de forma expositiva-dialogada e ao final uma atividade experimental em grupo foi realizada entre os acadêmicos. Para avaliar o alcance das intenções iniciais, foi aplicado um questionário após a atividade experimental. Foi possível verificar o engajamento dos licenciandos nas aulas enquanto materializavam as questões e conseguiam resolver os exercícios. 0 interesse despertou-se com o uso de materiais simples, mostrando ser possível motivar e contribuir na formação de futuros professores.

PALAVRAS-CHAVE: Centro de Massa; Ensino de Física; M aterialização de exercícios. 


\section{INTRODUÇÃO}

As dificuldades encontradas no processo de ensino e aprendizagem de Física, devido, entre outros fatores, à falta de investimentos na educação, são recorrentes e têm apresentados reflexos na formação dos professores e alunos, em especial os oriundos de instituições públicas.

0 ensino da Física, a nível médio, nas escolas brasileiras apresentam duas vertentes contraditórias. De um lado, a constatação de que se trata de uma área de conhecimento importante, de outro, a insatisfação diante dos resultados negativos obtidos com frequência, em relação à sua aprendizagem. A insatisfação revela que há problemas a serem enfrentados, tais como a necessidade de reverter um ensino centrado em procedimentos mecânicos, desprovidos de significados para o aluno. Sendo assim, para reverter-se tal situação, entende-se a necessidade de reformular objetivos, rever conteúdos e buscar metodologias de ensino compatíveis com a formação que hoje a sociedade requer (PCN, 2000, apud GOMES \& CASTILHO, 2010).

Além disso, a falta de prestígio da profissão docente tem levado à docência um grupo de profissionais com fraca bagagem cultural, em especial na área das Ciências Exatas (SALLA \& RATIER, 2009), pertencendo esses a um dos grupos que vem enfrentando maiores dificuldades no seu trabalho.

A literatura mostra que são grandes as dificuldades enfrentadas pelo professor. 0 excesso de alunos em sala de aula, o volume de conteúdo não condizente com a carga horária das disciplinas, falta de infraestrurura nas escolas, pouca receptividade por parte de alguns alunos em virtude de desinteresse e desmotivação. Quanto a desmotivação dos alunos, entende-se que esteja relacionada às suas deficiências em relação aos seus próprios conhecimentos prévios e/ou às dificuldades de materialização de seus conhecimentos pela não existência ou existência insuficiente de atividades de ensino práticas/ experimentais.

De uma ou outra forma, considera-se que esse cenário tem contribuído para dificultar, por parte dos alunos, a compreensão e apropriação de novos conhecimento, refletindo-se diretamente na capacidade de resolução de problemas mais comprexos. Nesse sentido, Silvério (2001) comenta sobre a existência de uma desmotivação dos alunos devido à dificuldade desses em realizar conexões entre o conhecimento científico, teórico e a prática. Não obstante, obsevam-se também problemas na própria formação do docente: didática, de conteúdos e/ou ambas.

Diante disso, entende-se que os professores universitários precisam buscar alternativas para auxiliar os alunos (FONTES, et al, 2016), repensando seus métodos no processo de ensino aprendizagem (CARVALHO, 2016), pois, dessa forma, poderão contribuir com melhorias na formação dos acadêmicos nos mais diversos cursos universitários do país, em especial os de licenciatura.

Assim, entende-se que é de extrema importância novas ações docentes no processo de ensino e aprendizagem, entre essas ações a de promover a motivação dos acadêmicos, em especial os dos cursos de licenciatura (FONTES, et al, 2017), pois se medidas não forem tomadas, os mesmos poderão apresentar desmotivados e com formação deficiente. 
Pesquisas na área de ensino e aprendizagem têm apresentado possíveis ações e experiências na tentativa de minimizar tais dificuldades. Entre tais ações está a construção de sequências didáticas que tem o potencial de trazer significado ao conhecimento, bem como propiciar para o professor-pesquisador caminhos para compreender como ocorre a apropriação do conhecimento. Pois, acredita-se que conhecimento se constrói a partir da identidade e a fomentação do espírito investigativo (GALAGOVSKY e M UÑOZ, 2002).

Neste sentido, acredita-se que as atividades experimentais apresentam perspectiva de promover diversas possibilidades para 0 aprendizado significativo. As atividades experimentais contribuem para a aplicação dos conhecimentos de teóricos em atividades práticas, contribuem também para o desenvolvimento da capacidade cognitiva e do raciocínio científico, favorecendo o trabalho em equipe por meio da discussão, da divisão de tarefas e da argumentação lógica. Espera-se com isso, um progresso na formação de conceitos e no desenvolvimento do aluno.

Assim, objetiva-se, nesse trabalho, apresentar relato de uma pesquisa desenvolvida em um ambiente de ensino e aprendizagem, aplicada na disciplina de Física 1, em uma turma de acadêmicos do curso de licenciatura em Química. Nesse processo, buscou-se, a partir de um conjunto de atividades propostas, verificar os efeitos dessas atividades no processo de ensino e aprendizagem do conteúdo de ensino Centro de Massa (CM ). 0 trabalho se desenvolveu seguindo os pressupostos da metodologia Pesquisa-Ação. A análise dos efeitos cognitivos da atividade empregada ocorreu tendo como referencial os pressupostos da Teoria da Aprendizagem Significativa (TAS).

\section{PRESSUPOSTOS DE ENSINO E APRENDIZAGEM}

Acredita-se que uma aprendizagem em que haja identidade e logo significado seja mais efetiva do ponto de vista da aprendizagem. A aprendizagem significativa é como um processo por meio do qual uma nova informação se relaciona com um aspecto especificamente relevante da estrutura de conhecimento do indivíduo, ou seja, um processo que envolva a interação entre a nova informação com uma estrutura de conhecimento já adquirida pelo aprendiz (AUSUBEL, NOVAK e HANESIAN,1980).

Nessa perspectiva, se a aprendizagem de novas informações não apresentar interação ou tiver pouca interação com conceitos relevantes e específicos da estrutura cognitiva do indivíduo, ocorre aprendizagem mecânica, pois, nesse caso, as novas informações são armazenadas de maneira arbitrária e literal (AUSUBEL, NOVAK e HANESIAN, 1980; GALAGOVSKY e M UÑOZ, 2002).

Para Ausubel, Novak e Galesian (1980), a escolha do mecanismo e do material de ensino pode ser um diferencial potencializador para da aprendizagem significativa. Deste modo, o material e o método de ensino a serem trabalhados com os alunos devem ser potencialmente significativos. E, como consequência, a proposta de um material diferenciado em sala pode contribuir para maior motivação e envolvimento desses alunos em sala de aula.

Página | 54

Para facilitar a aprendizagem significativa, Ausubel propõe a diferenciação progressiva, onde ideias mais amplas e gerais são abordadas antes de conceitos mais específicos. Ele sugere que é mais fácil aprender um conteúdo mais geral e 
depois partir para suas especificidades. Denomina-se reconciliação integrativa o evento/processo no qual os conceitos já existentes na estrutura cognitiva se reorganizam e adquirem novos significados frente às novas informações incorporadas (AUSUBEL, NOVAK e HALESIAN, 1980).

Entre as diferentes estratégias, Silva e Beltran Nunez (2002, p.1199, apud MACHADO, V., 2009), indicam a possibilidade do uso de trabalhos experimentais.

\begin{abstract}
Quando se resolve um problema nas aulas de ciências, ainda que seja para a construção de um conceito, isso possibilita estruturar algum tipo de trabalho experimental que contribua com o desenvolvimento de habilidades e atitudes. 0 trabalho experimental deixa de ser uma simples comprovação de conhecimentos, para se transformar numa atividade motivadora, que pode ajudar os alunos a desenvolverem atitudes, questionarem suas próprias idéias, construírem metodologias que revelem 0 caráter contraditório do conhecimento para comprovarem suas hipóteses, em função de um determinado fundamento teórico.
\end{abstract}

Dessa forma, acredita-se que, a relação afetiva entre professor e aluno, associada a um material potencialmente significativo tem como objetivo potencializar o aluno para que o mesmo esteja predisposto ao aprendizado e que a sua construção de conhecimento venha a ocorrer significativamente. Deste modo, essa pesquisa tem suas motivações e influencia teórica voltada a aprendizagem significativa.

\title{
CENTRO DE MASSA
}

0 centro de massa de um sistema de partículas é o ponto que se move como se toda a massa do sistema estivesse concentrada nesse ponto e todas as forças externas estivessem aplicadas nesse ponto. Mesmo quando um corpo gira ou vibra, existe um ponto, chamado centro de massa (CM), que se desloca da mesma maneira que se deslocaria uma única partícula, de mesma massa deste corpo e sujeita ao mesmo sistema de forças que ele. Ainda que o sistema não seja um corpo rígido, mas um conjunto de partículas pode ser definido para ele um CM, ou seja, o centro de massa não precisa estar contido em um corpo (HALLIDAY, RESNICK e WALKER, 2008).

No dia a dia, é comum deparar-se com situações em que o conceito de centro de massa esteja envolvido, por exemplo, com o movimento de qualquer corpo rígido, ou sistema de partículas, qualquer que seja a sua complexidade, podendo ser descrito em termos do movimento do $\mathrm{CM}$ e do movimento das partículas individuais do sistema em relação ao CM. Ele é uma característica intrínseca do corpo e independe de fatores externos.

Assim, entende ser importante diferenciar de antemão os conceitos de centro geométrico (CG) de uma figura de um objeto e de centro de massa (CM) de um objeto. 0 conceito de centro geométrico normalmente é aplicado às figuras planas e corresponde ao centroide dessa figura conforme mostrado na Figura 1.

No conceito de centro de massa é levada em consideração a massa de cada elemento que compõe o objeto. No caso específico onde o objeto apresenta distribuição de massa homogênea e uniforme (com a mesma densidade em todos os pontos) os pontos de CG e CM são coincidentes. 
Figura 1- Centro de gravidade e centro de massa para objetos homogêneos submetidos a um campo gravitacional constante
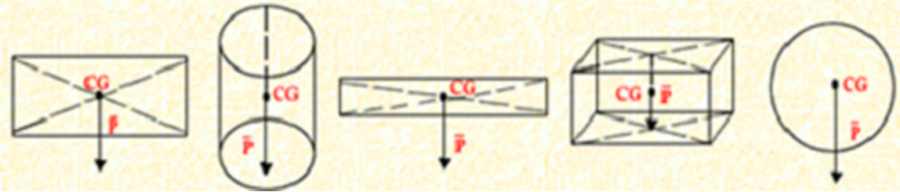

Fonte: Ramalho Junior, Ferraro e Soares (2007, p.400).

Para um Sistema de Partículas, em uma dimensão, composto de duas partículas de massas $m_{1}$ e $m_{2}$, massa total $M$ e que ocupam as posições $x_{1}$ e $x_{2}$ em relação à origem (Figura 2), pode-se definir a posição $x_{c m}$ (eq. 1) do centro de massa para um sistema da seguinte forma:

$$
x_{c m}=\frac{m_{1} x_{1}+m_{2} x_{2}}{M}
$$

Em que M representa a massa total das partículas do sistema.

Figura 2- Sistema de partículas

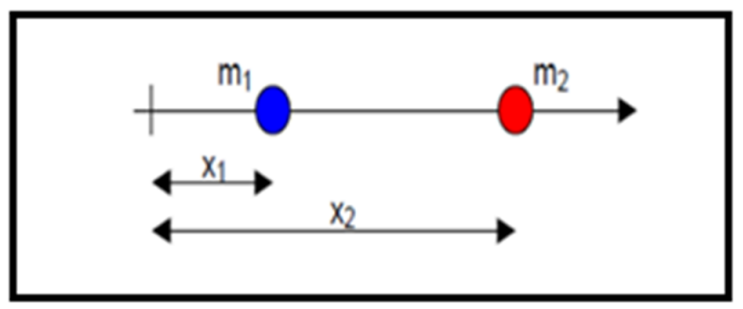

Fonte: autores.

Para um Sistema, contendo N partículas, dispostos ao longo de uma linha reta a coordenada $\mathrm{x}$ do centro de massa se define por:

$$
x_{c m}=\frac{m_{1} x_{1}+m_{2} x_{2}+m_{3} x_{3}+\ldots+m_{N} x_{N}}{M}=\frac{1}{M} \sum_{i=1}^{N} m_{i} x_{i}
$$

na qual $M=\sum_{i=1}^{N} m_{i}$ é a massa total do sistema.

Equações semelhantes definem as coordenadas y e $z$ do centro de massa, para um sistema com duas ou três dimensões.

$$
\begin{aligned}
& y_{c m}=\frac{m_{1} y_{1}+m_{2} y_{2}+m_{3} y_{3}+\ldots+m_{N} y_{N}}{M}=\frac{1}{M} \sum_{i=1}^{N} m_{i} y_{i} \\
& z_{c m}=\frac{m_{1} z_{1}+m_{2} z_{2}+m_{3} z_{3}+\ldots+m_{N} z_{N}}{M}=\frac{1}{M} \sum_{i=1}^{N} m_{i} z_{i}
\end{aligned}
$$

Considerando a notação vetorial, $\vec{r}_{i}=x_{i} \hat{i}+y_{i} \hat{j}+z_{i} \hat{k}$ define o vetor posição da $i-$ ésima partícula, onde o índice $\mathbf{i}$ identifica a partícula e $\hat{\mathbf{i}}, \hat{\mathbf{j}}$ e $\hat{\mathbf{k}}$ são vetores 
unitários. As três equações escalares (2, 3 e 4) podem ser substituídas por uma única equação vetorial (5), para a obtenção do vetor posição do centro de massa,

$$
\vec{r}_{c m}=\frac{m_{1} \vec{r}_{1}+m_{2} \vec{r}_{2}+m_{3} \vec{r}_{3}+\ldots}{M}=\frac{1}{M} \sum_{i=1}^{N} m_{i} \vec{r}_{i}
$$

Já os corpos contínuos/ maciços, em função de conterem muitas partículas (átomos), podem ser aproximados por uma distribuição contínua de massa. As partículas, nesse caso, tornam-se elementos infinitesimais de massa [dm], sendo que as somas das equações (2, 3 e 4$)$ geram as integrais que definem as coordenadas do CM para cada um dos eixos do espaço 3D conforme mostrado nas equações (6).

$$
x_{c m}=\frac{1}{M} \int x d m, \quad y_{c m}=\frac{1}{M} \int y d m, \quad z_{c m}=\frac{1}{M} \int z d m
$$

Para descrever a relação entre os elementos de massa [dm] e suas respectivas posições, $x$, y ou $z$, consideram-se corpos homogêneos, que possuem massa específica ( $\rho$ ) uniforme, ou seja, a massa específica é a mesma para todos os elementos do corpo e para o corpo como um todo, assim representa-se:

$$
\rho=\frac{d m}{d v}=\frac{M}{V} \Leftrightarrow d m=\frac{M}{V} d v
$$

Substituindo dm, nas equações acima, para $x_{c m}, y_{c m}$ e $z_{c m}$ teremos:

$$
\mathrm{x}_{\mathrm{cm}}=\frac{1}{\mathrm{~V}} \int \mathrm{xdv}, \quad \mathrm{y}_{\mathrm{cm}}=\frac{1}{\mathrm{~V}} \int \mathrm{ydv}, \quad \mathrm{z}_{\mathrm{cm}}=\frac{1}{\mathrm{~V}} \int \mathrm{zdv}
$$

No caso particular do objeto ser homogêneo e apresentar um ponto, uma reta ou um plano de simetria, o cálculo das integrais acima pode ser simplificado, pois, nesse caso, o CM está no ponto, linha ou plano de simetria.

\section{METODOLOGIA}

Apresentam-se, nesse trabalho, relatos de uma investigação aplicada, baseada no método da pesquisa-ação. Buscou-se promover uma ação em que os professores que atuam em formação de acadêmicos do curso de química tivessem a oportunidade de participar de uma atividade diferenciada em sala de aula.

Investigações realizadas no âmbito do ensino de ciências apresentam a pesquisa-ação como opção metodológica adotada em processos realizados na formação inicial de licenciandos e em programas de formação continuada de professores (MONCEAU, 2005). Kemmis \& Wilkinson (2002) apresentam a pesquisa-ação no contexto educacional, não apenas como meio de mudança e desenvolvimento profissional. Em suas palavras:

[...] a pesquisa-ação participativa tenta ajudar, orientar as pessoas a investigarem e a mudarem suas realidades sociais e educacionais por meio da mudança de algumas das práticas que constituem suas realidades vividas. Em educação, a pesquisa-ação participativa pode ser utilizada como meio de desenvolvimento profissional, melhorando currículos ou solucionando 
problemas em uma variedade de situações e trabalho (KEMMIS \& WILKINSON, 2002, p. 44-45).

Considerando tais motivações esse trabalho se estabeleceu como uma pesquisa-ação, sendo desenvolvido durante a disciplina Física 1, na forma de atividade prática como componente curricular (PCC), com vinte alunos do curso de Licenciatura em Química, em uma Universidade Pública na cidade de Campo Mourão.

0 trabalho foi dividido em duas etapas: inicialmente, durante aula teórica, foi apresentado o conteúdo de forma a identificar o conhecimento prévio dos alunos sobre 0 assunto e, na sequência, foi trabalhado de forma expositivadialogada, com demonstrações de equações e resoluções de exercícios.

$\mathrm{Na}$ segunda etapa, no laboratório de Física 1, foi realizada a atividade prática, onde os alunos foram divididos em cinco grupos de quatro componentes cada, denominados de $A, B, C, D$ e $E$, e receberam a tarefa de reconstruir os exercícios/exemplos previamente selecionados do livro, usando os materiais concretos de baixo custo e o roteiro elaborado pela professora.

Os materiais utilizados para essa atividade são de fácil obtenção: bolinha de gude, palitos de madeira, balança, papel quadriculado e massa de modelar.

Para a realização desta sequência didática foram utilizadas seis aulas, e verificou-se que o tempo foi suficiente de modo a fazer com que os alunos compreendessem os conceitos fundamentais sobre o tema abordado. Durante a última aula, foi aplicado um questionário estruturado que visava avaliar as contribuições e limitações da sequência didática aplicada neste trabalho.

A contextualização do conteúdo CM foi realizada em primeiro momento, durante a explicação do conteúdo em sala de aula e nas discussões após a realização da atividade prática.

A Sequência Didática, de acordo com Pais (2002), "é formada por certo número de aulas planejadas e analisadas previamente com a finalidade de observar situações de aprendizagem, envolvendo os conceitos previstos na pesquisa didática".

Ao ser aplicada de maneira organizada, em uma perspectiva sociocultural bem direcionada e com objetivos claros, essa estratégia pedagógica pode contribuir de forma significativa no processo de ensino e aprendizagem, minimizando as tensões de um ensino descontextualizado e da ação desconexa das áreas de ensino no ambiente escolar (GUIM ARÃES \& GIORDAN, 2011).

Ainda é preciso ressaltar que o professor precisa estar envolvido com o seu próprio trabalho, pois o professor é, por excelência, o principal agente motivador e precisa estar determinado, ter compromisso pessoal com a sua formação e com a formação do discente, demonstrando dedicação, entusiasmo e amor no que faz. 0 professor deve ser 0 agente que busca mobilizar a energia interna do aluno para um propósito direcionado (CARVALHO, 2016).

Em uma proposta de trabalho voltada a uma aprendizagem significativa em uma perspectiva metodológica como a pesquisa-ação é preciso que o professor esteja engajado nas intervenções em sala, propondo de modo participativo ações que contribuam para a aprendizagem. 


\section{DESENVOLVIMENTO DAS ATIVIDADES}

\section{ATIVIDADE 1}

$\mathrm{Na}$ atividade 1, solicitou-se aos alunos que reproduzissem um sistema de partículas unidimensional, baseado nas informações contidas na Figura 3a, a fim de obter a localização do CM em relação à origem.

Observou-se no decorrer do processo que os alunos não encontraram dificuldades para realizar essa atividade. Iniciaram pela realização das medidas das massas $\left(m_{1}\right.$ e $\left.m_{2}\right)$ das bolas de gude em uma balança digital, e após posicionaram-nas sobre um papel quadriculado, separadas por uma distância d, onde $\mathrm{m}_{1}$ estava localizada na origem do sistema (Figura $\mathbf{3 b}$ ).

Figura 3- a) A figura mostra duas partículas de massas $m_{1}$ e $m_{2}$ que estão separadas por uma distância d; b) Reprodução do sistema

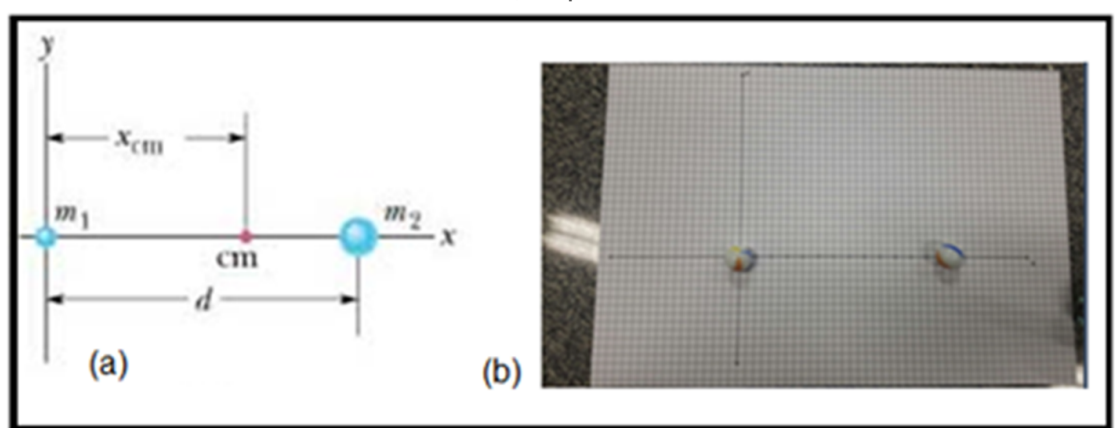

Fonte: a) Halliday, Resnick e Walker (2008, p. 219); b) alunos do Grupo B.

Por meio da representação gráfica e do uso da equação (9), a posição do CM foi obtida pelos estudantes. Com o desenvolvimento dessa questão, os alunos puderam compreender do porque da equação (1) se reduzir à equação (9), situação em que uma das massas está localizada na origem do sistema adotado.

$$
x_{c m}=\frac{m_{1} \cdot 0+m_{2} x_{2}}{m_{1}+m_{2}}=\frac{m_{2} \cdot x_{2}}{m_{1}+m_{2}}
$$

\section{ATIVIDADE 2}

Na sequência, a atividade 2 , indicada na Figura $4 a$, propôs que a $m_{1}$ fosse deslocada para a esquerda, aproximando-a da massa $m_{2}$ e que o CM fosse calculado em relação à origem.

Assim como na primeira atividade, observou-se que os alunos não apresentaram dificuldades na realização, sendo que ambas servem para apresentar ao aluno o conceito de centro de massa de um sistema de partículas em uma dimensão de uma maneira prática e atraente.

Pelos cálculos, realizados com a equação (1), os alunos puderam perceber que houve uma mudança na posição do centro de massa do sistema, tendo seu valor aumentado, indicando uma aproximação da partícula 1 em relação a posição da partícula 2 . 
Figura 4- (a) A partícula $m_{1}$ foi deslocada, aproximando-se de $m_{2}$; b) Reprodução do sistema

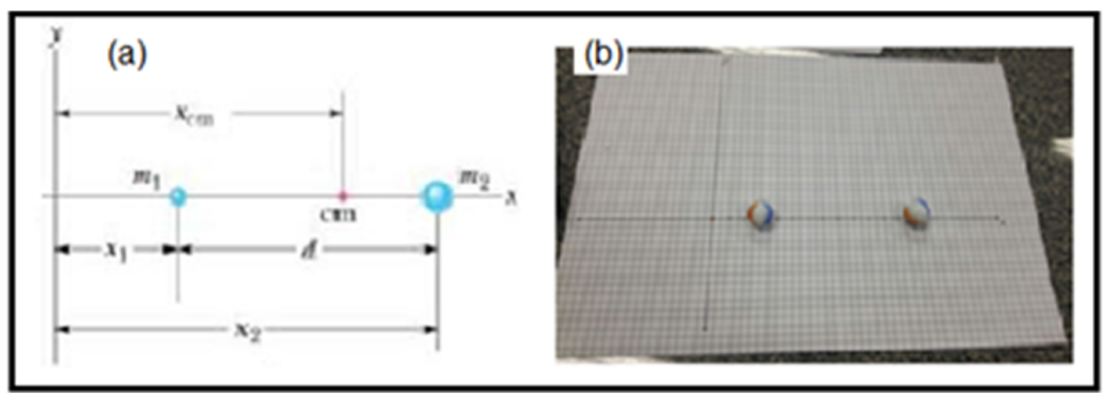

Fonte: a) Halliday, Resnick e Walker (2008, p. 219); b) alunos do Grupo B.

\section{ATIVIDADE 3}

Para um sistema de partículas em duas dimensões, foi adaptada uma terceira atividade tendo como modelos o exemplo da Figura 5a, e o exercício 2 (pg. 247) do livro e foi dividida em duas partes. A primeira parte propunha a obtenção do CM de um sistema contendo três partículas de massas conhecidas que formaram um triângulo equilátero de lado conhecido.

A atividade foi reproduzida posicionando três esferas sobre o papel quadriculado (Figura 5b), de modo a formar um triângulo equilátero, considerando uma das massas na origem do sistema.

As coordenadas do CM foram facilmente determinadas graficamente e matematicamente utilizando as equações (2) e (3), sendo que na sequência, também foram apresentadas em termos de vetores unitários, utilizando para isso a equação (5). As coordenadas x e y de cada uma das partículas foram obtidas a partir da Figura 5b e transferidas para o Quadro 1.

Quadro 1- Valores encontrados pelo grupo C, para as massas das esferas e suas coordenadas de CM

\begin{tabular}{|c|c|c|c|}
\hline Esfera & $\begin{array}{c}\text { Massa } \\
(\mathbf{K g})\end{array}$ & $\begin{array}{c}\text { Coordenada X } \\
(\mathbf{c m})\end{array}$ & $\begin{array}{c}\text { Coordenada Y } \\
(\mathbf{c m})\end{array}$ \\
\hline $\mathbf{1}$ & 0,00569 & 0 & 0 \\
\hline $\mathbf{2}$ & 0,00543 & 150 & 0 \\
\hline $\mathbf{3}$ & 0,00524 & 75 & 130 \\
\hline
\end{tabular}

Fonte: alunos do Grupo C.

Neste caso, a escala adotada pelo grupo foi a que cada em divisão do papel quadriculado teve equivalência a $10 \mathrm{~cm}$. Os resultados obtidos pelo grupo $\mathrm{C}$ utilizando as equações (2 e (3) foram $X_{\mathrm{cm}}=73,81 \mathrm{~cm}$ e $Y_{\mathrm{cm}}=41,64 \mathrm{~cm}$, respectivamente, que são as coordenadas do CM do triângulo.

Em termos de vetores unitários, o vetor posição é $\overrightarrow{r_{c m}}=(73,81 \hat{\imath}+41,64 \hat{\jmath})$ $\mathrm{cm}$. Realizando as atividades conforme a ordem apresentada neste trabalho, os alunos puderam construir os conceitos do CM, compreendendo quando devem calcular mais de uma coordenada do CM . 
Figura 5- (a) Três partículas de massas $m_{1}, m_{2}$ e $m_{3}$ formam um triângulo equilátero de lado a; b) Adaptação do exemplo 9-1 e c) Adaptação do exercício 2 com o aumento da

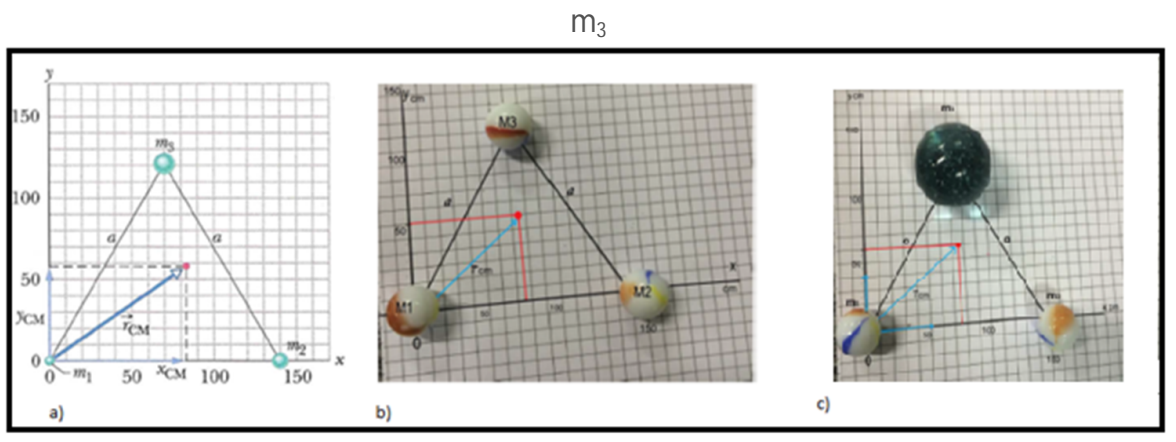

Fonte: a) Halliday, Resnick e Walker, (2008, p. 221); b e c) alunos do Grupo C.

Na segunda parte da terceira atividade propôs-se que fosse aumentada a massa $m_{3}$ e que se analisasse 0 que aconteceria com o CM. Os grupos refizeram a atividade, substituindo a massa 3 por outra maior e puderam visualizar (Figura 5c) que o CM se aproximou simetricamente de $m_{3}$.

Entende-se que 0 desenvolvimento da atividade contribuiu para 0 entendimento do porque o centro de massa mudou, quando se aumentou a massa. Foi verificada uma variação desprezível em relação à posição $x$ do $\mathrm{CM}$ e uma variação significativa ao logo do eixo y, fazendo com que o novo CM se aproximasse de $m_{3}$

\section{ATIVIDADE 4}

A quarta atividade solicitou aos alunos que identificassem as coordenadas do CM do sistema de três barras finas de comprimento $L$, em forma de $U$ invertido (Figura 6a).

Primeiramente os alunos reproduziram a figura (Figura $6 \mathbf{6 b}$ ) sobre o papel quadriculado e marcaram o CM de cada barra. Em seguida, uniram os três CM, formando no papel um triângulo. Assim, puderam visualizar, por simetria, que 0 CM estava mais próximo da barra horizontal (segunda barra) e por cálculos puderam obter sua localização exata.

Figura 6- a) Três barras finas em forma de U; b) Materialização da figura a; c) Quadrado; d) Comparativo do CM das Figuras b e c

Com base na Figura $\mathbf{6 b}$ os estudantes extraíram as coordenadas do CM de cada palito e após, calcularam as coordenadas do CM do sistema. Neste sentido, 
os estudantes poderiam ter utilizado as equações (6), mas como observaram que, a união do CM de cada barra formava o triangulo equilátero, optaram em calcular as coordenadas do CM do triângulo equilátero, conforme feito na Figura 5, usando as equações (2) e (3).

A solução gráfica para essa questão pode ser obtida encontrando o centro geométrico do triângulo obtido a partir dos CM das barras. Os grupos não tiveram dificuldade alguma em resolver esta questão, visto que na terceira atividade já haviam trabalhado no CM do triângulo equilátero.

A fim de explorar um pouco mais a atividade, foi solicitado que inserissem um quarto palito (barra), formando um quadrado oco (Figura 6c), de modo a obterem graficamente e matematicamente $0 \mathrm{CM}$ da figura.

Os dados das coordenadas X e Y de cada barra, extraídos da Figura $6 c$ estão descritos no Quadro 2.

Quadro 2- Dados extraídos da figura 6c

\begin{tabular}{|l|c|c|c|}
\hline Barra & $\begin{array}{c}\text { Massa } \\
(\mathbf{k g})\end{array}$ & $\begin{array}{c}\text { Coordenada x } \\
(\mathbf{c m})\end{array}$ & $\begin{array}{c}\text { Coordenada y } \\
\mathbf{( c m})\end{array}$ \\
\hline $\mathbf{1}$ & 0,0001 & 0 & 3,25 \\
\hline $\mathbf{2}$ & 0,0001 & 3,25 & 6,5 \\
\hline $\mathbf{3}$ & 0,0001 & 6,5 & 3,25 \\
\hline $\mathbf{4}$ & 0,0001 & 3,25 & 0 \\
\hline
\end{tabular}

Fonte: alunos do Grupo B.

De posse dos dados do Quadro 2 e das equações (2) e (3), os estudantes obtiveram o CM do sistema onde puderam visualizar que o centro geométrico (CG) do quadrado, coincide com o CM .

Ao comparar os resultados da posição do CM das Figuras 6b (figura $U$ invertido) e $6 c$ (quadrado), percebeu-se que houve um deslocamento na coordenada $Y$.

Isso ocorreu quando transformou a figura $U$ invertido (Figura 6 b) em um quadrado (Figura $\mathbf{6 c}$ ), indicando que a posição do $\mathrm{CM}$ anteriormente estava mais próxima da barra 2 (horizontal), migrando para o centro, conforme representado pelas bolas azul (Fig. 6b) e amarela (Fig. 6c) na Figura 6d.

Na sequência, solicitou-se que fossem colocadas pequenas bolas feitas com massa de modelar, possuindo a mesma massa, nas extremidades dos palitos, afim de uní-los conforme se visualiza na Figura $\mathbf{6 d}$, com o intuito de compreender melhor a localização do CM. Os alunos puderam perceber que o CM continua situado no centro geométrico do quadrado.

\section{ATIVIDADE 5}

Seguindo o roteiro, solicitou-se que os alunos reproduzissem o problema da Figura 7, no qual deveriam construir sobre um papel quadriculado, um cubo oco de palitinhos usando massa de modelar de modo a obter as coordenadas do CM. 
Figura 7- Reprodução do cubo

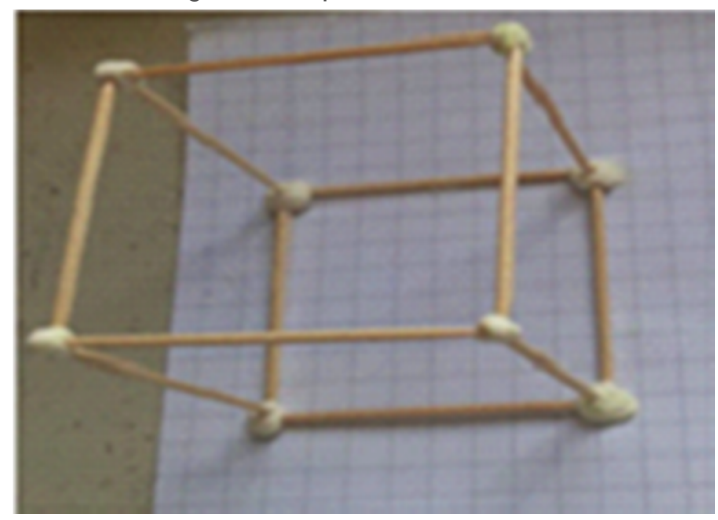

Fonte: alunos do Grupo A.

As massas foram consideradas desprezíveis, sendo utilizadas apenas para unir os palitos. Os dados obtidos dessa atividade estão descritos no Quadro 3.

Quadro 3- Valores encontrados para as coordenadas do CM

\begin{tabular}{|c|c|c|c|}
\hline Aresta & $\begin{array}{c}\text { Coordenada X } \\
\text { (cm) }\end{array}$ & $\begin{array}{c}\text { Coordenada Y } \\
\text { (cm) }\end{array}$ & $\begin{array}{c}\text { Coordenada Z } \\
\text { (cm) }\end{array}$ \\
\hline $\mathbf{1}$ & 3,25 & 0 & 0 \\
\hline $\mathbf{2}$ & 0 & 3,25 & 0 \\
\hline $\mathbf{3}$ & 3,25 & 6,5 & 0 \\
\hline $\mathbf{4}$ & 6,5 & 3,25 & 0 \\
\hline $\mathbf{5}$ & 3,25 & 0 & 6,5 \\
\hline $\mathbf{6}$ & 0 & 3,25 & 6,5 \\
\hline $\mathbf{7}$ & 3,25 & 6,5 & 6,5 \\
\hline $\mathbf{8}$ & 6,5 & 3,25 & 6,5 \\
\hline $\mathbf{9}$ & 6,5 & 0 & 3,25 \\
\hline $\mathbf{1 0}$ & 0 & 0 & 3,25 \\
\hline $\mathbf{1 1}$ & 0 & 6,5 & 3,25 \\
\hline $\mathbf{1 2}$ & 6,5 & 6,5 & 3,25 \\
\hline
\end{tabular}

Fonte: alunos do Grupo A.

Com o uso das equações (2, 3 e 4), obtiveram as coordenadas do CM para um sistema em três dimensões, os quais foram: $X_{c m}=Y_{c m}=Z_{c m}=3,2 \mathrm{~cm}$. Os resultados obtidos apresentam uma boa aproximação entre os valores experimentais e teóricos, de modo que o valore esperado seria $X_{\mathrm{cm}}=Y_{\mathrm{cm}}=Z_{\mathrm{cm}}=$ $3,25 \mathrm{~cm}$.

\section{ATIVIDADE 6}

Dando sequência, propôs-se que cada face do cubo fosse preenchida com massa de modelar para a obtenção da posição do CM. Após encontrados os valores das áreas e as coordenadas de cada face do cubo obtidas da figura os dados foram inseridos no Quadro 4. Com o uso das equações 2, 3 e 4, os alunos obtiveram $X \mathrm{~cm}=\mathrm{Ycm}=\mathrm{Zcm}=3,25 \mathrm{~cm}$. 
Quadro 4- Valores encontrados para as áreas e as coordenadas do CM

\begin{tabular}{|r|c|c|c|c|}
\hline Face & $\begin{array}{c}\text { Área } \\
\left(\mathbf{c m}^{\mathbf{2}} \mathbf{)}\right.\end{array}$ & $\begin{array}{c}\text { Coordenada X } \\
(\mathbf{c m})\end{array}$ & $\begin{array}{c}\text { Coordenada } \mathbf{Y} \\
(\mathbf{c m})\end{array}$ & $\begin{array}{c}\text { Coordenada Z } \\
(\mathbf{c m})\end{array}$ \\
\hline $\mathbf{1}$ & 42,25 & 6,5 & 3,25 & 3,25 \\
\hline $\mathbf{2}$ & 42,25 & 0 & 3,25 & 3,25 \\
\hline $\mathbf{3}$ & 42,25 & 3,25 & 6,5 & 3,25 \\
\hline $\mathbf{4}$ & 42,25 & 3,25 & 0 & 3,25 \\
\hline $\mathbf{5}$ & 42,25 & 3,25 & 3,25 & 0 \\
\hline $\mathbf{6}$ & 42,25 & 3,25 & 3,25 & 6,5 \\
\hline
\end{tabular}

Fonte: alunos do Grupo A.

Com o desenvolvimento das atividades perceberam também que poderiam ter utilizado a equação (5) e que o mesmo valor do CM poderia ser obtido por meio de métodos gráficos, pois corresponde ao centro geométrico do cubo.

\section{ATIVIDADE 7}

A seguir, propôs-se na sétima atividade uma situação de determinação do CM de uma figura que entende-se exigir dos alunos a compreensão de todas as outras atividades realizadas anteriormente. Propôs-se, então, uma situação adaptada do problema 6 do livro (HALLIDAY, RESNICK e WALKER, 2008, p. 247) na qual foi considerado o mesmo cubo da atividade anterior, porém, somente com cinco faces preenchidas e uma vazada, ou seja, um problema conhecido por a caixa sem tampa.

Os dados utilizados nesta atividade foram os mesmos do Quadro 4, desprezando a sexta face. Os cálculos para a obtenção das coordenadas do CM foram realizados com o uso das equações ( 2,3 e 4), substituindo a aresta pela área, obtendo $X_{c m}=Y_{c m}=3,2 \mathrm{~cm}$ e $Z_{c m}=2,6 \mathrm{~cm}$.

Outro modo de resolver essa questão pode ocorrer pelo uso da equação (5). Determina-se o CM de cada um dos cinco lados da caixa e em seguida, ao considerar a caixa como um sistema de massas pontuais, para então encontrar 0 CM da caixa sem tampa. 0 CM de cada lado da caixa é obtido por:

$$
\begin{aligned}
& \overrightarrow{r_{1}}=\frac{a}{2} \hat{\imath}+\frac{a}{2} \hat{k} ; \overrightarrow{r_{2}}=a \hat{\imath}+\frac{a}{2} \hat{\jmath}+\frac{a}{2} \hat{k} ; \overrightarrow{r_{3}}=\frac{a}{2} \hat{\imath}+a \hat{\jmath}+\frac{a}{2} \hat{k} ; \overrightarrow{r_{4}}=\frac{a}{2} \hat{\jmath}+\frac{a}{2} \hat{k} ; \\
& \overrightarrow{r_{5}}=\frac{a}{2} \hat{\imath}+\frac{a}{2} \hat{\jmath}
\end{aligned}
$$

Substituindo o valor da aresta, $a=6,5 \mathrm{~cm}$, e aplicando a equação (5), foi obtido o vetor posição do $\mathrm{CM}: \overrightarrow{\mathrm{r}_{\mathrm{cm}}}=(3,2 \hat{\imath}+3,2 \hat{\jmath}+2,6 \hat{\mathrm{k}}) \mathrm{cm}$. Comparando a atividade [6] com a [7], percebeu-se uma pequena diferença no valor das coordenadas. Isso se deve em função da retirada de uma face na atividade 7 (tampa).

\section{ATIVIDADE 8}

A última atividade foi adaptada a partir das notas de aula do professor DANTAS (2016), sendo a situação apresentada por uma chapa em L de material homogêneo mostrada na Figura 8a. Dividiu-se a chapa em três partes retangulares, as quais foram reproduzidas utilizando massa de modelar, sobre 0 
papel quadriculado, objetivando indicar o CM de cada uma das partes, comprovando matematicamente os valores.

Figura 8- a) Placa em forma de L, composta por três materiais distintos; b) Reprodução elaborada pelo grupo B

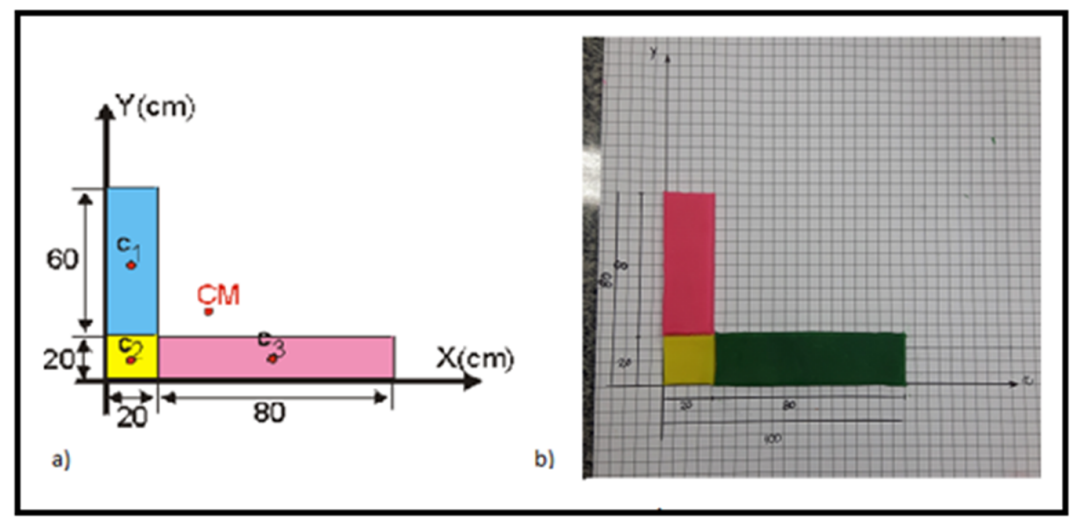

Fonte: a) Dantas (2016); b) alunos do Grupo B.

A Figura $\mathbf{8 b}$ mostra as coordenadas de cada parte da placa encontradas pelos alunos, valores apresentados no Quadro 5.

Quadro 5- Valores encontrados para as áreas e as coordenadas do CM

\begin{tabular}{|c|c|c|c|}
\hline Cor & $\begin{array}{c}\text { Área } \\
\left(\mathbf{c m}^{2}\right)\end{array}$ & $\begin{array}{c}\text { Coordenada } \mathrm{x}_{\mathrm{cm}} \\
(\mathbf{c m})\end{array}$ & $\begin{array}{c}\text { Coordenada } \mathrm{y}_{\mathrm{cm}} \\
(\mathbf{c m})\end{array}$ \\
\hline Rosa & 1200 & 10 & 50 \\
\hline Amarelo & 400 & 10 & 10 \\
\hline Verde & 1600 & 60 & 10 \\
\hline
\end{tabular}

Fonte: alunos do Grupo B.

Para a obtenção da posição do CM do sistema, foram utilizadas as equações (2) e (3) fazendo uma substituição da massa pela área da figura, onde obtiveram facilmente os valores $X_{c m}=35 \mathrm{~cm}$ e $Y_{c m}=25 \mathrm{~cm}$. Por meio dessa atividade os alunos puderam visualizar uma situação em que o CM fica localizado fora do corpo.

De um modo geral, as questões acima discutidas ajudaram a trabalhar na formação dos conceitos, transformação de unidades, escalas, gráficos, instrumentos de medida, o cálculo das áreas e volumes das figuras geométricas além da compreensão das equações sobre CM apresentadas no livro texto.

Por meio do uso do roteiro descrito acima, percebeu-se que obedecendo a uma evolução natural do grau de dificuldade das questões, a sequência didática foi ordenada de modo a calcular o centro de massa de um sistema de partículas em uma dimensão, evoluindo na sequência para duas, três dimensões e depois para um corpo rígido (placa).

Durante a realização da atividade, foi observado que os grupos conseguiam desempenhar as atividades propostas, demonstrando motivação e compreensão dos conceitos e fórmulas. De acordo com Ausubel, Novak e Hanesian (1980), quando existe significado, ou seja, motivação e identidade, o ambiente se torna propício para a transformação da informação em conhecimento. E isso, pôde ser observado pelo desempenho entre os participantes. 
Após a realização da atividade, os alunos responderam a um questionário, sendo este aplicado com o intuito de confirmar se tais indícios caracterizavam se a experimentação pôde contribuir efetivamente no processo ensinoaprendizagem ao aluno.

A primeira questão visava avaliar o conceito de CM. Durante a análise das respostas, percebeu-se que a atividade conseguiu fazer com que os alunos compreendessem sobre a localização do CM de vários objetos e formas. "[...] estruturas vazadas, ocas ou não geométricas (ocas ou sólidas), podem possuir centro de massa localizado em uma região que não pertença ao corpo, portanto o CM não está restrito à parte sólida do corpo" (alunos do Grupo A).

Observa-se na resposta dada que os alunos desse grupo conseguiram visualizar que nem sempre o CM está localizado no interior da figura.

$\mathrm{Na}$ segunda questão, sobre a atividade 2 , solicitou-se aos alunos que avaliassem a influência sobre o CM se, no sistema, fosse inserido um palito entre as bolas de massa iguais. 0 grupo B relatou que: "Não mudaria a posição do centro de massa se a barra for uniforme".

Quatro grupos conseguiram visualizar que a inserção de uma barra fina (palito) entre as bolas não influenciaria o valor da posição do CM. Somente 0 grupo $\mathrm{E}$ se confundiu, respondendo que $0 \mathrm{CM}$ das bolas se deslocaria aproximando-se do CM do palito, sem se ater que o CM do palito coincidia com 0 CM das bolas, ou seja, não há deslocamento. Mas, após as discussões, os alunos desse grupo puderam corrigir o raciocínio, causado pela falta de atenção na interpretação da questão.

Por fim, na última questão foi solicitado aos alunos que fizessem um comentário sobre a prática.

\begin{abstract}
Ao estudarmos esse assunto utilizando o livro Halliday, nos deparamos com algumas dificuldades, das quais, a maioria estava relacionada à falta de interpretação do assunto. Para conseguir calcular o CM, não basta apenas conhecera teoria e dominar fórmulas, mas sim, "enxergar" seu real efeito no corpo. Por isso essa prática acabou com nossas dúvidas. Achamos a prática trabalhosa. Foi preciso anotar bastante coisa, medir, cortar, montar. Em suma, todo o grupo precisou se mobilizar para ajudar na execução do trabalho. Contudo, apesar de trabalhosa, achamos muito divertido trabalhar com as massinhas de modelar e palitos, podemos dizer que foi possível dar uma descontraída com a atividade. 0 trabalho ajudou a concretizar a teoria que havíamos estudado em sala e deixou o assunto mais "visível" além de possibilitar uma boa interação entre o grupo e nos permitir aprender brincando (alunos do Grupo D).
\end{abstract}

Com base no relato acima, entende-se ser possível afirmar que a atividade prática resultou em um fator motivador para o estudo do $\mathrm{CM}$, tendo em vista a participação ativa e o comprometimento de cada membro do grupo. Pode-se ainda verificar que a sequencia de atividades propostas foram significativas, pois aproximaram os conceitos teóricos à realizade momentânea dos alunos por meio das atividades práticas realizadas.

Segundo Carvalho (1989), um dos aspectos fundamentais no ensino de Física Página | 66 é conhecer como os alunos percebem e compreendem o mundo físico que os cerca. É a partir destes conhecimentos que os professores de Física podem construir um ensino mais eficiente e motivador. 


\section{CONSIDERAÇÕES FINAIS}

Considerando que as atividades experimentais abordadas neste trabalho foram trabalhadas após a interação incial dos alunos com o conteúdo exposto pelo livro texto e, tomando por base os comentários dos grupos de alunos durante a realização das atividades práticas, foi constatado que essas atividades possibilitaram a interação com os conteúdos abordados anteriormente, incluindo os subsunçores relacionados à geometria básica. Assim, entende-se que o processo desenvolvido cumpriu com uma das etapas indicadas por Ausubel para potencializar a aprendizazem significativa.

Neste cenário, o mecanismo de ensino que envolveu atividades participativas e em grupos, forneceu uma forma mais atraente de tratar 0 conteúdo, permitindo que os alunos participassem de uma forma mais ativa das discuções, compartilhando o conhecimento com os demais integrantes, também potencializando a aprendizagem significativa.

Verificou-se a escassez de kits comercias que pudessem dar suporte para elaboração de atividades práticas que envolvesse o conteúdo de CM. Na maioria dos kits comerciais encontrados, os conceitos de CM estão inseridos em contextos mais amplos, como por exemplo, no estudo dos pêndulos físicos, mais especificamente no teorema dos eixos paralelos. Desta forma, entende-se que a sequência didática apresentada neste trabalho apresenta-se como uma alternativa viável para a superação da falta de recursos materiais, pois utiliza de materiais de baixo custo para aplicação das atividades.

Por fim, tendo a certeza que não foram esgotados os assuntos pertinentes ao conteúdo abordado neste trabalho, espera-se que este trabal ho possa fornecer uma alternativa diferenciada para o trabalho dos conteúdos relacionados ao CM para outros professores. Assim, espera-se que o trabalho aqui apresentado seja capaz de fornecer auxílio aos colegas docentes ao mostrar uma maneira atraente para trabalhar possíveis dificuldades dos alunos em resgatar conhecimentos prévios. $E$, nesse caso, numa turma de acadêmicos do curso de licenciatura em Química, dar a eles motivação, enquanto futuros professores, para que possam entender de maneira mais ampla e prática o conteúdo trabalhado. 


\title{
Materialization of Questions Related to Center of Mass: an Activity Applied to Undergraduate
}

\begin{abstract}
Through this article, it is presented an action developed in the discipline of Physics I, with undergraduate students in a Chemistry course of a Public University in up-country Paraná. With this activity, one attempted to contribute to minimize the difficulty of undergraduates in interpreting exercises in the adopted textbook, through the materialization of exercises on the theme "Center of Mass". Initially the content was introduced in order to identify prior knowledge. Afterwards, an expository-dialogued work was conducted, and, at finally, an experimental group activity was carried out among the undergraduates. At the conclusion of the research, a questionnaire was applied to evaluate the scope of initial intentions. It was possible to verify the commitment of the undergraduates in the classes while materializing the questions, being able to solve the exercises. The interest was promoted by the use of simple materials, showing that it is possible to motivate and contribute to the training of future teachers.
\end{abstract}

KEYWORDS: Center of Mass; Physics Teachin; Reconstruction of Exercises. 


\title{
Materialización de cuestiones relacionadas al Centro de Masa: una actividad aplicada a un curso de graduación
}

\begin{abstract}
RESUMEN
En este artículo se presenta una investigación acción que se desarrolló con estudiantes de licenciatura en Química de una universidad pública del estado de Paraná, en la asignatura Física 1. Se ha buscado con esa actividad ayuda para minimizar la dificultad de interpretación académica del libro texto que se ha adoptado, a través de la materialización de ejercicios con la temática Centro de Masa. Inicialmente se introdujo el contenido con el fin de identificar los conocimientos previos. Tras el trabajo expositivo dialogado, se hizo una actividad experimental en grupo con los académicos y finalmente la encuesta. Se aplicó un cuestionario para evaluar el alcance de las intenciones iniciales. Ha sido posible verificar la participación de los académicos en las clases, mientras se materializaron problemas y ellos se sentían capaces de resolver los ejercicios. Ha sido despertado el interés por el uso de materiales simples, enseñando que ello puede motivar y contribuir a la formación de los futuros docentes.
\end{abstract}

PALABRAS CLAVE: Centro de masa; Enseñanza de Física; Materialización de ejercicios. 


\section{REFERÊNCIAS}

AUSUBEL, D.P., NOVAK, J.D. \& HANESIAN, H. Psicologia Educacional. Rio de Janeiro: Interamericana. Tradução da segunda edição de Educational psychology: A cognitive view, 1980.

CARVALHO, A. M. P. Física: Proposta para um ensino construtivista, São Paulo: EPU, 1989.

CARVALHO, A.S. O papel do professor no ensino superior. Formação \& ação docente, v.8, n.1, p. 2-4, 2016.

GOMES, J. C.; CASTILHO, W. S. Uma visão de como à física é ensinada na escola brasileira, e a experimentação como estratégia para mudar essa realidade. Anais eletrônicos - 1a Jornada de iniciação científica e extensão do IFTO, 2010.

DANTAS, V. B. Centro de massa. Disponível em: বhttps:// www.yumpu.com/pt/ document/ view/14513996/integrais-do-centro-demassapara-transparencia-htmlpdf3pdf-ufersa/3>Acesso em 15 mar.2016.

FONTES, A. S.; CONEGLIAN, D. R.; CANOVAS, D. P. S.; BATISTA, M. C. Tem física no cachimbo da vovó? Anais eletrônico - XXII Simpósio Nacional de Ensino de Física. Sociedade Brasileira de Física. São Carlos, 2017.

FONTES, A. S.; RAM OS, F. P.; SCHEREK, R. C.; CARGNIN, C. Jogos adaptados para 0 ensino de física, Ensino, Saúde e Ambiente, v. 9, n. 3, p. 226-248, 2016.

GALAGOVSKY, L. R.; MUÑOZ, J. C. La distancia entre aprender palabras e aprender er conceptos: el entramado de palavras - concepto (EPC) como un Nuevo instrumento para la investigatón, Ensenãnza de las ciências, v. 20, n. 1, p. 29-45, 2002.

GUIM ARÃES, Y.A.F. \& GIORDAN, M. Instrumento para construção e validação de sequências didáticas em um curso a distância de formação continuada de professores (In:VIII ENPEC -Encontro nacional de pesquisa em educação em ciências, Campinas, 2011).

HALLIDAY, D.; RESNICK, R.; WALKER, J. Fundamentos da Física, v. 1, M ecânica, 8a Ed., Rio de Janeiro: LTC, 2008.

KEM MIS, S. E WILKINSON, M. Pesquisa-ação participativa e o estudo da prática. In: Pereira, Júlio E. Diniz e Zeichner, Kenneth M. A pesquisa na formação e no trabalho docente. 1 a edição. Belo Horizonte: Autêntica, 2002.

MONCEAU, G. Transformar as práticas para conhecê-las: pesquisa-ação e profissionalização docente. In: Educação e Pesquisa, v.31, n.3, set./dez.São Paulo: FEUSP, 2005.

PAIS, L. C. Didática da Matemática: uma análise da influência francesa, Belo Horizonte: Autêntica, 2002.

RAMALHO JUNIOR, F.; FERRARO, N. G.; SOARES, P. A. T. Os fundamentos da Física, 9a. Ed. rev. e ampl., São Paulo: M oderna, 2007.

SALLA, F.; RATIER, R. Por que tão poucos querem ser professor? Atratividade da carreira docente no Brasil. Revista Nova Escola, Fundação Victor Civita, Edição Especial, 2009. Disponível em: 〈http://www.fvc.org.br/pdf/atratividadecarreira.pdf >Acesso em: 07 de nov. 2016. 
SILVA, S. F.; BELTRAN NUNEZ, I. 0 ensino por problemas e trabalho experimental dos estudantes: reflexões teórico-metodológicas. Quím. Nova, São Paulo, v. 25, n. 6b, dez. 2002. In: MACHADO, V. Problemas geradores de discussões: uma proposta para a disciplina de física nos cursos de engenharia. Dissertação. Programa de Pós-Graduação em Ensino de Ciência, Universidade Tecnológica Federal do Paraná. Ponta Grossa, 2009.

SILVÉRIO, A. A. As dificuldades no ensino/aprendizagem da física. Monografia. Programa de Pós-graduação em Ensino de Física. Centro de Ciências Físicas e Matemáticas. Universidade Federal de Santa Catarina. Departamento de Física. Florianópolis, 2001.

Recebido: 08 de abril de 2017

Aprovado: 04 de maio de 2017.

DOI:

Como citar: FONTES, A.S.; RAMOS, F.P. e DEIMLING, C.V. , Materialização de questões relacionadas a Centro de Massa: uma atividade aplicada a licenciandos, Revista Brasileira de Física Tecnológica Aplicada, Ponta Grossa, v. 4, n.1, p. 52-71, mai./ jun. 2017.

Contato: Adriana da Silva Fontes: asfontes@utfpr.edu.br

Direito autoral: Este artigo está licenciado sob os termos da Licença Creative Commons-Atribuição 4.0 Internacional.

\section{(c) (1)}

